\title{
High-energy electron experiments (HEP) aboard the ERG (Arase) satellite
}

\author{
Takefumi Mitani ${ }^{1 *}$, Takeshi Takashima' ${ }^{1}$ Satoshi Kasahara² ${ }^{2}$ Wataru Miyake ${ }^{3}$ and Masafumi Hirahara ${ }^{4}$
}

\begin{abstract}
This paper reports the design, calibration, and operation of high-energy electron experiments (HEP) aboard the exploration of energization and radiation in geospace (ERG) satellite. HEP detects $70 \mathrm{keV}-2 \mathrm{MeV}$ electrons and generates a three-dimensional velocity distribution for these electrons in every period of the satellite's rotation. Electrons are detected by two instruments, namely HEP-L and HEP-H, which differ in their geometric factor (G-factor) and range of energies they detect. HEP-L detects $70 \mathrm{keV}-1 \mathrm{MeV}$ electrons and its $\mathrm{G}$-factor is $9.3 \times 10^{-4} \mathrm{~cm}^{2} \mathrm{sr}$ at maximum, while HEP-H observes $0.7-2 \mathrm{MeV}$ electrons and its G-factor is $9.3 \times 10^{-3} \mathrm{~cm}^{2} \mathrm{sr}$ at maximum. The instruments utilize silicon strip detectors and application-specific integrated circuits to readout the incident charge signal from each strip. Before the launch, we calibrated the detectors by measuring the energy spectra of all strips using $\gamma$-ray sources. To evaluate the overall performance of the HEP instruments, we measured the energy spectra and angular responses with electron beams. After HEP was first put into operation, on February 2, 2017, it was demonstrated that the instruments performed normally. HEP began its exploratory observations with regard to energization and radiation in geospace in late March 2017. The initial results of the in-orbit observations are introduced briefly in this paper.
\end{abstract}

Keywords: ERG, Arase, Radiation belts, High-energy electrons

\section{Introduction}

The mechanisms by which electrons are accelerated in geospace are a key research area in solar-terrestrial plasma physics. Relativistic electrons are trapped in the outer Van Allen radiation belt. The electron flux is known to decrease rapidly during the main phase of the magnetic storms, and then increases during the storms' recovery phase (Baker et al. 1986; Nagai 1988; Reeves et al. 2003). The processes causing flux variation must be investigated with comprehensive in situ observations. Particle acceleration occurs in various environments around astronomical objects such as supernova remnants and pulsar-wind nebulae (Makishima 1999). Moreover, the Earth's radiation belts offer a unique opportunity to observe these particles and waves in situ. Armed with an understanding of the acceleration mechanism in geospace, some insight might be gained into the phenomena of electron acceleration in other astronomical objects.

\footnotetext{
*Correspondence: mitani@planeta.sci.isas.jaxa.jp

${ }^{1}$ Institute of Space and Astronautical Science, Japan Aerospace

Exploration Agency, Sagamihara 252-5210, Kanagawa, Japan

Full list of author information is available at the end of the article
}

The exploration of energization and radiation in geospace (ERG) project explores the acceleration, transportation, and loss of relativistic electrons in the radiation belts and the dynamics of storms in geospace (Miyoshi et al. 2012; Miyoshi et al. in review). To gain a detailed understanding of the acceleration and transport processes, the electrons must be observed over a wide range of energies and electromagnetic fields with a wide range of frequencies. The experiment observes a wide range of geospace particles, including the warm electrons, hot electrons of the plasma sheet, and sub-relativistic and relativistic electrons of the radiation belts. The satellite uses four instruments, namely LEP-e, MEP-e, HEP, and $\mathrm{XEP}$, in order to measure electrons over this wide range of energies (Kazama et al. 2017; Kasahara et al. 2018a; Higashio et al. in review). The high-energy electron experiments (HEP) onboard the ERG satellite detects $70 \mathrm{keV}-2 \mathrm{MeV}$ electrons and generates a three-dimensional velocity distribution of electrons for every period of the satellite's spin. This energy range covers relativistic electrons and their seed electrons. The full suite of instruments aboard the ERG observes a wide range of 
electrons. Three additional devices have been installed: XEP (Higashio et al. in review) detects electrons with energies of 0.4-20 MeV, MEP-e (Kasahara et al. 2018a) detects electrons with energies of 7-87 keV, and LEP-e (Kazama et al. 2017) detects electrons with energies of $17 \mathrm{eV}-20 \mathrm{keV}$. In MEP-e, the electrons are energy-filtered by an electrostatic analyzer and the maximum detected energy is $87 \mathrm{keV}$. To allow for continuous energy coverage, the lower detection range of HEP overlaps with the maximum MEP-e energy.

The ERG satellite, which is also known as 'Arase', was launched from the Uchinoura Space Center at 11:00 on December 20, 2016, by using the Epsilon launch vehicle. The spacecraft attained an orbit with an apogee and perigee altitude of approximately 32,000 and $400 \mathrm{~km}$, respectively. This allowed the satellite to record observations over the entire extent of the radiation belts. After all the instruments and functions were successfully checked, the scientific observations commenced in late March 2017.

This paper describes the design of the HEP instruments, prelaunch testing, and initial results of the inorbit observations.

\section{Instrument design}

HEP consists of two types of telescopes, namely HEP-L and HEP-H, which have different geometric factors (G-factor) and energy ranges. As summarized in Table 1, HEP-L observes $70 \mathrm{keV}-1.0 \mathrm{MeV}$ electrons, and the G-factor of its three detector modules is $9.3 \times 10^{-4} \mathrm{~cm}^{2}$ sr. HEP-H observes $0.7-2.0 \mathrm{MeV}$, and its G-factor is $9.3 \times 10^{-3} \mathrm{~cm}^{2}$ sr. These G-factors are numerically calculated and account only for the geometrical information regarding the collimator and detectors. Since the effective area of the detectors can be selected via parameters in the readout electronics, the above G-factor is a maximum value. If we assume that the maximum electron flux in the satellite's orbit is $10^{9} \times(\mathrm{E} /[\mathrm{keV}])^{-2}$, then, the total count rate is estimated to be $4.4 \mathrm{kHz}$ above $70 \mathrm{keV}$, and $440 \mathrm{~Hz}$ above $0.7 \mathrm{MeV}$ by using the G-factor of the HEP-L module, for which the total count of electrons above $0.7 \mathrm{MeV}$ in each eight-second rotation period is estimated to be $3.5 \times 10^{3}$ over $4 \pi$ steradians. To detect more electrons at $0.7 \mathrm{MeV}$, the geometric factor of HEP-H is designed to be ten times larger than that of HEP-L. A photograph of the HEP flight model is shown in Fig. 1. Three HEP-L and three HEP-H modules are housed in the hexagonal cylinder, and the electronics boards are housed in the black box. Each of the three sides of the hexagonal cylinder has two slits, through which the electrons enter the instrument. Figure 2a illustrates the HEP's mounting onto the ERG satellite and marks its fields of view as gray areas corresponding to approximately $60^{\circ} \times 10^{\circ}$. The HEP instrument is mounted on the panel normal to the $+X$ axis of the ERG satellite. As the satellite rotates around the $\mathrm{Z}_{\mathrm{sc}}$-axis, HEP covers $4 \pi$ steradians. As shown in Fig. $2 \mathrm{~b}$, each module has a $60^{\circ}$ field of view in the elevation angle, and each one is divided into five channels, such that one channel corresponds to $12^{\circ}$. HEP-L and HEP-H consist of three pinhole cameras, and each camera consists of a mechanical collimator, stacked silicon semiconductor detectors, and application-specific integrated circuits (ASICs) to readout the signal. HEP-H has a larger collimator opening angle in order to attain a larger G-factor than that of HEP-L. Additionally, HEP-H utilizes more detectors to detect higher-energy electrons. Because the electrode of the silicon detector is segmented, the position at which an incident electron interacts can only be determined in

Table 1 HEP performance and specifications. Resolution is indicated by full-width at half maximum (FWHM)

\begin{tabular}{|c|c|c|}
\hline Parameter & HEP-L & HEP-H \\
\hline Energy range & $70 \mathrm{keV}-1.0 \mathrm{MeV}$ & $0.7-2.0 \mathrm{MeV}$ \\
\hline Energy resolution $(\triangle E / E)$ & $11 \%$ at $300 \mathrm{keV}, 18 \%$ at $750 \mathrm{keV}$ & $17 \%$ at $750 \mathrm{keV}, 12 \%$ at $1.2 \mathrm{MeV}$ \\
\hline $\begin{array}{l}\text { Energy binning of onboard } \\
\text { histogram }^{a}\end{array}$ & $\begin{array}{l}70,100,124,153,188,230,280340,412,499,605,730,850,990 \\
1400, \text { and } 1800(\mathrm{keV})\end{array}$ & $\begin{array}{l}100,153,230,340,412,499,605,730,850,990 \\
1200,1400,1600,1800, \text { and } 2000(\mathrm{keV})\end{array}$ \\
\hline Field of view & $\begin{array}{l}10^{\circ} \text { (Azimuth) } \times 60^{\circ} \text { (Elevation) for a module } \\
10^{\circ} \text { (Azimuth) } \times 180^{\circ} \text { (Elevation) for three modules }\end{array}$ & \\
\hline Angular resolution & $4^{\circ} \pm 1^{\circ}$ & $15^{\circ} \pm 3^{\circ}$ \\
\hline Geometric factor & $\begin{array}{l}3.1 \times 10^{-4} \mathrm{~cm}^{2} \mathrm{sr} \text { (one module) } \\
9.3 \times 10^{-4} \mathrm{~cm}^{2} \mathrm{sr} \text { (three modules) }\end{array}$ & $\begin{array}{l}3.1 \times 10^{-3} \mathrm{~cm}^{2} \mathrm{sr} \text { (one module) } \\
9.3 \times 10^{-3} \mathrm{~cm}^{2} \mathrm{sr} \text { (three modules) }\end{array}$ \\
\hline Time resolution & $\begin{array}{l}8 \mathrm{~s} \text { per full } 3-\mathrm{D} \text { distribution function (for normal spin period of } 8 \mathrm{~s} \text { ) } \\
\text { (15 histograms are generated for a } 1 / 16 \text { th of spin period) }\end{array}$ & \\
\hline Flux dynamic range & $10^{4}-10^{7} / \mathrm{cm}^{2} / \mathrm{s} / \mathrm{sr}$ & $10^{3}-10^{6} / \mathrm{cm}^{2} / \mathrm{s} / \mathrm{sr}$ \\
\hline Mass & $6.8 \mathrm{~kg}$ & \\
\hline Power & $17.6 \mathrm{~W}$ & \\
\hline
\end{tabular}

${ }^{a}$ Hatched values are for calibration purposes. All values can be changed as onboard software parameters with commands issued from the ground 


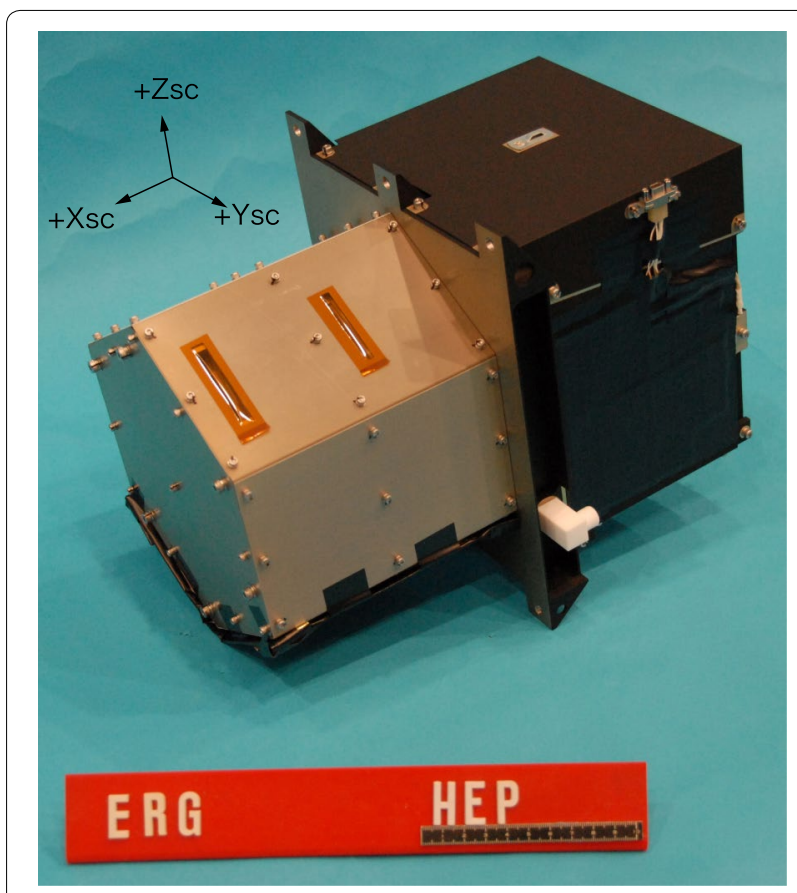

Fig. 1 Photograph of HEP flight model. The marked axes are the satellite coordinates shown in Fig. 2. In the left part of the figure, it can be seen that the hexagonal cylinder houses three HEP-L modules and three HEP-H modules. Electronics boards are housed in the black box shown in the right part of the figure. Each of the three sides of the hexagonal cylinder includes two cutouts through which the electrons enter the HEP-L/HEP-H sensors

one dimension. Each HEP-L module utilizes four silicon strip detectors (SSDs), while each HEP-H module has eight SSDs. The collimator design and SSD placement are illustrated in Fig. 3, and Table 2 lists the sizes of the SSDs.

\section{Signal processing}

Electrons passing through the collimator interact with the SSDs and deposit energy inside them. The interaction positions and amount of deposited energy are measured by the SSDs in conjunction with the readout electronics. A functional block diagram for the instrument is shown in Fig. 4. The SSD modules in HEP-L and HEP-H are independently controlled by two control boards that include field programmable gate arrays (FPGAs) and point-of-load (POL) DC-DC converters to power the ASICs. The central processing unit (CPU) board is part of the ERG mission network (Takashima et al. 2018) and utilizes the SpaceWire data link to receive command packets and send telemetry data. The CPU board collects the histogram data from the control boards, in addition to the data collected to monitor the instrument's condition. The SSD bias voltage is supplied by a high-voltage-power supply board that includes two independent high-voltage DC-DC converters, whose power and reference voltage are controlled by the control boards. HEP also has an interface for the software wave-particle interaction analyzer (S-WPIA) clock signal, which is used to determine the electron incidence timing and generate packets for the wave-particle analyzer software included in the mission data processor (Hikishima et al. 2018). We describe each of these functional blocks in further detail below.

\section{Stacked silicon strip detector module}

The total silicon thicknesses for the SSDs in HEP-L and HEP-H are 1.85 and $4.25 \mathrm{~mm}$, respectively. These silicon thicknesses correspond to the range of $850 \mathrm{keV}$ and $1.7 \mathrm{MeV}$ electrons, respectively, according to the ESTAR web database of electron stopping powers and ranges, which is maintained by the National Institutes of Standards and Technology (NIST). The incident direction of the detected particles is determined from the position of the interaction at the first layer and the geometrical position of the collimator. To determine the interaction position, HEP uses SSDs, whose electrodes are subdivided into closely spaced strips. As summarized in Table 2, HEP-L consists of one 50- $\mu$ m-thick SSD and three $600-\mu$ m-thick SSDs, while HEP-H consists of one $50-\mu \mathrm{m}$-thick SSD and seven $600-\mu \mathrm{m}$-thick SSDs. All SSDs were manufactured by HAMAMATSU Photonics. $\mathrm{K}$. K. The full depletion voltage of the silicon wafers, from which the detectors were made, was 18 and $80 \mathrm{~V}$ for the 50 - and $600-\mu \mathrm{m}$-thick SSDs, respectively. To choose the operation bias voltages for the SSDs, we measured the spectra by using a radioisotope while changing the bias voltage. We chose 20 and $200 \mathrm{~V}$ as the operation bias voltage for the 50- and 600- $\mu \mathrm{m}$-thick SSDs, respectively.

To block the protons, an $\mathrm{Al}$ sheet is placed in front of the $50-\mu \mathrm{m}$-thick SSD. The Al shield for HEP-L is $12.5 \mu \mathrm{m}$ thick. This is the typical length traveled by a $0.9-\mathrm{MeV}$ proton in $\mathrm{Al}$, based on the PSTAR proton-range table provided by NIST. Therefore, the protons with lower energy are stopped in the $\mathrm{Al}$ sheet. To reduce the incidence of low-energy electrons in $\mathrm{HEP}-\mathrm{H}$, a $300-\mu \mathrm{m}$-thick $\mathrm{Al}$ sheet is placed in front of the stacked SSDs. This thickness corresponds to the typical distance traveled by the $6.5-\mathrm{MeV}$ protons or the $0.3-\mathrm{MeV}$ electrons in $\mathrm{Al}$. The $50-\mu \mathrm{m}$-thick SSD is also utilized to separate the electrons and protons penetrating the $\mathrm{Al}$ sheet. To illustrate this concept, Fig. 5 shows the results of a simple detector simulation with the Geant4 toolkit, which simulates the passage of particles through matter. Figure 5a shows the differential flux of the electrons and protons at $L=3$ based on the AE8MAX/AP8MAX model (see the web page 'AE-8/ AP-8 Radiation Belt Models'). The simulation irradiates the electrons or protons according to the energy distribution onto the stacked SSD shielded by an $\mathrm{Al}$ sheet 


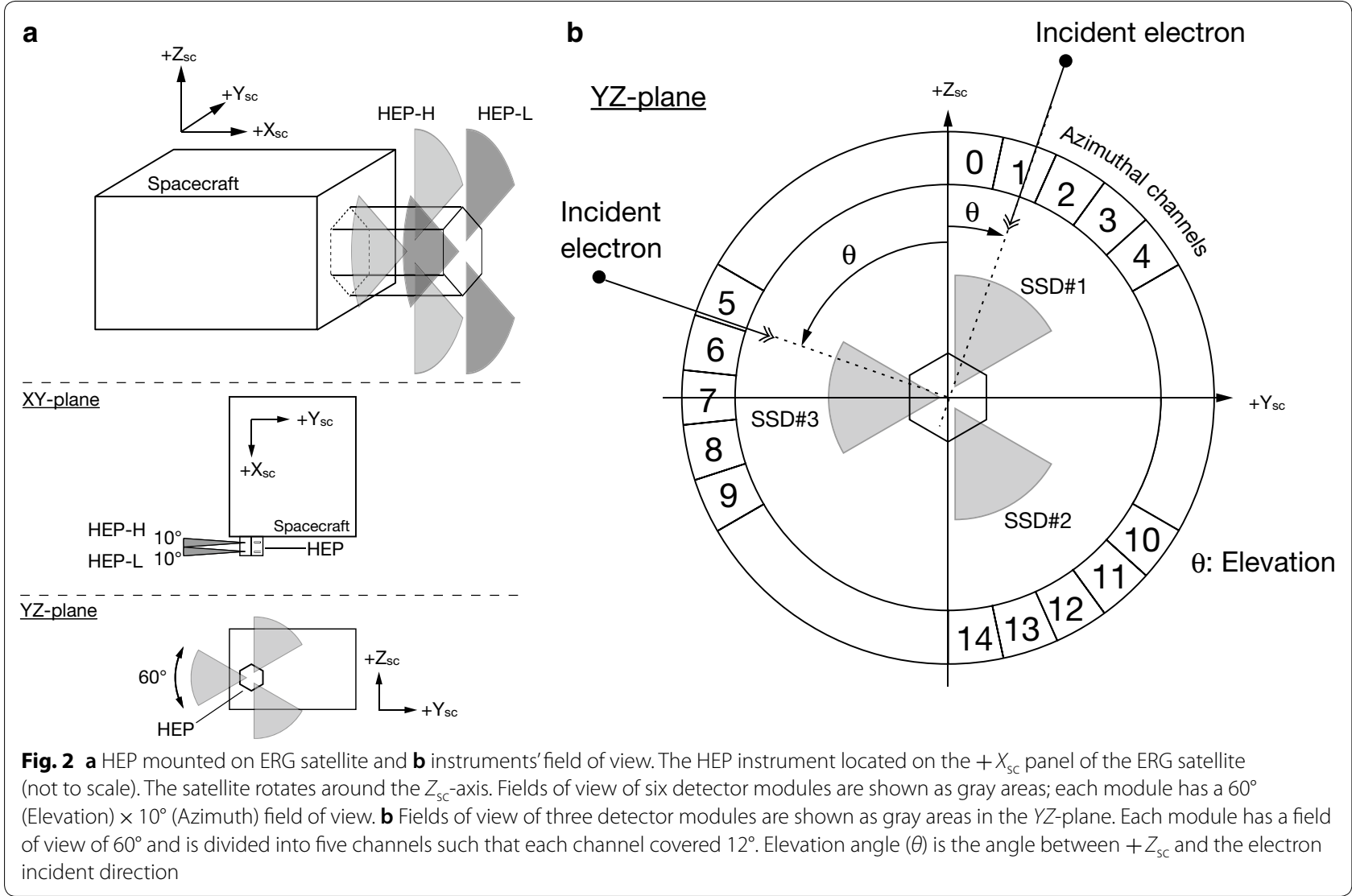

with the same thickness as that of the $\mathrm{Al}$ sheet installed on HEP-L. Figure $5 \mathrm{~b}$ shows the simulated energy spectra to be measured by the $50-\mu \mathrm{m}$-thick SSD. In this case, the counts above the energy of $140 \mathrm{keV}$ are generally caused by protons. Therefore, proton contamination can be reduced by ignoring the events for which the energy at the thin SSD is larger than $140 \mathrm{keV}$. However, the energy threshold depends on the relative distributions of electrons and protons. By taking these considerations into account, HEP is equipped with a function that assessed an incident particle as a proton if the deposited energy at the first layer is higher than a certain threshold and if the energy deposited in the second layer approximated zero. This function can be disabled, and the threshold energy $E$ th can be set remotely.

The position resolution is determined by the pitch of the strips in the $50-\mu \mathrm{m}$-thick SSD. To estimate the number of strips that will detect an incident charge, we simulate the interaction of electrons with the detector by using the Geant 4 toolkit. In the simulation, a monoenergetic pencil beam of electrons is irradiated onto the detector at normal incidence from the collimator input. 100 - and $500-\mathrm{keV}$ beams are simulated to test HEP-L, and $750-\mathrm{keV}$ and $1.5-\mathrm{MeV}$ beams are simulated to test
HEP-H. The standard deviations of the strip ID in HEP-L, which detects the maximum energy in the first layer, are 8.2 strips for the $100-\mathrm{keV}$ electrons and 2.9 strips for the $500-\mathrm{keV}$ electrons, while those in HEP-H are 7.5 strips for the $750-\mathrm{keV}$ electrons and 7.1 strips for the $1.5-\mathrm{MeV}$ electrons. When calculated by the distance between the collimator and the detector, which is indicated by $\mathrm{R}$ in Fig. 3, these values correspond to the incidence angles of $9.7^{\circ}, 3.5^{\circ}, 7.9^{\circ}$, and $7.4^{\circ}$, respectively.

The detection signal from each strip is read out by an ASIC called VATA460.3. We have developed VATA460.3 in collaboration with IDEAS in Norway. Its development has carried out based on the designs of high-energy particle sensors aboard the BepiColombo MMO (Saito et al. 2010) and the soft gamma-ray detector aboard the Astro$\mathrm{H}$ (Watanabe et al. 2014). As shown in Fig. 6, VATA460.3 consists of 32-channel low-noise MOS amplifiers and Wilkinson 10-bit analog-to-digital converters (ADCs). The ASIC's power consumption is $0.6 \mathrm{~mW} / \mathrm{ch}$. Each channel included a charge-sensitive preamplifier, a fast CR-RC shaping amplifier followed by a discriminator that generated a trigger signal for the readout sequence, and a slow CR-RC shaping amplifier followed by a sample-andhold circuit for pulse-height analysis. The preamplifiers 


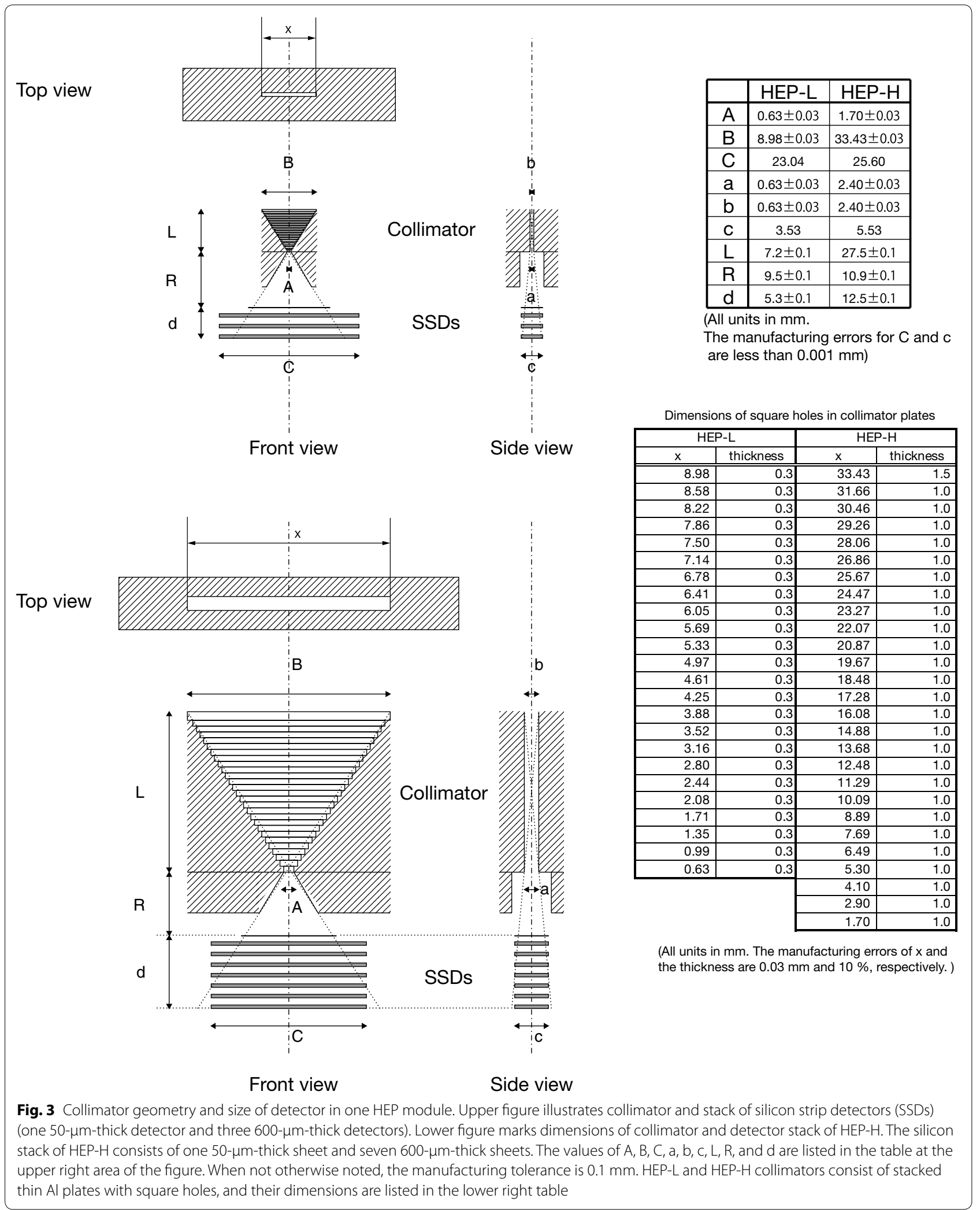


Table 2 Sizes of silicon strip detectors used in HEP

\begin{tabular}{llllll}
\hline & Layer $(\boldsymbol{\mu m})$ & Thickness $(\boldsymbol{\mu m})$ & Strip pitch $^{\mathbf{a}}(\boldsymbol{\mu m})$ & Strip length $^{\mathbf{a}}(\mathbf{m m})$ & Number of strips \\
\hline HEP-L & Al sheet & $12.5 \pm 2.5$ & & & 3.53 \\
& 1st layer & $50 \pm 10$ & 200 & 3.53 & 67 \\
2nd-4th layer & $600 \pm 10$ & 360 & & 78 \\
HEP-H & $300 \pm 30$ & $50 \pm 10$ & 200 & 5.53 & 64 \\
& Al sheet & $600 \pm 10$ & 400 & 5.53 & \\
\hline
\end{tabular}

a The manufacturing errors for the strip pitch and the strip length are less than $\pm 1 \mu \mathrm{m}$

accept a maximum input of $90 \mathrm{fC}$, which corresponds to $2 \mathrm{MeV}$ in $\mathrm{Si}$. The fast and slow shaping times are 0.6 and $2 \mu \mathrm{s}$, respectively, and each one can be tuned by using the control registers. The Wilkinson ADC converts the held pulse height to a digital value in less than $100 \mu \mathrm{s}$. The digital data from the 32 channels are multiplexed and displayed on a serialized digital interface. The strips in the first layer are read out by three ASICs, and those in the lower layers are read out by two ASICs each. Therefore, 9 and 17 ASICs are used in one SSD module of the HEP-L and HEP-H, respectively. To tune the bias parameters and channel logic, each ASIC has a 520-bit control register. The tunable parameters include the shaping time and global threshold for each chip, and information on whether the trigger output for each channel is enabled. With these registers, we can change which channels issues the trigger signals from the ground. This means that the effective detection area can be reduced

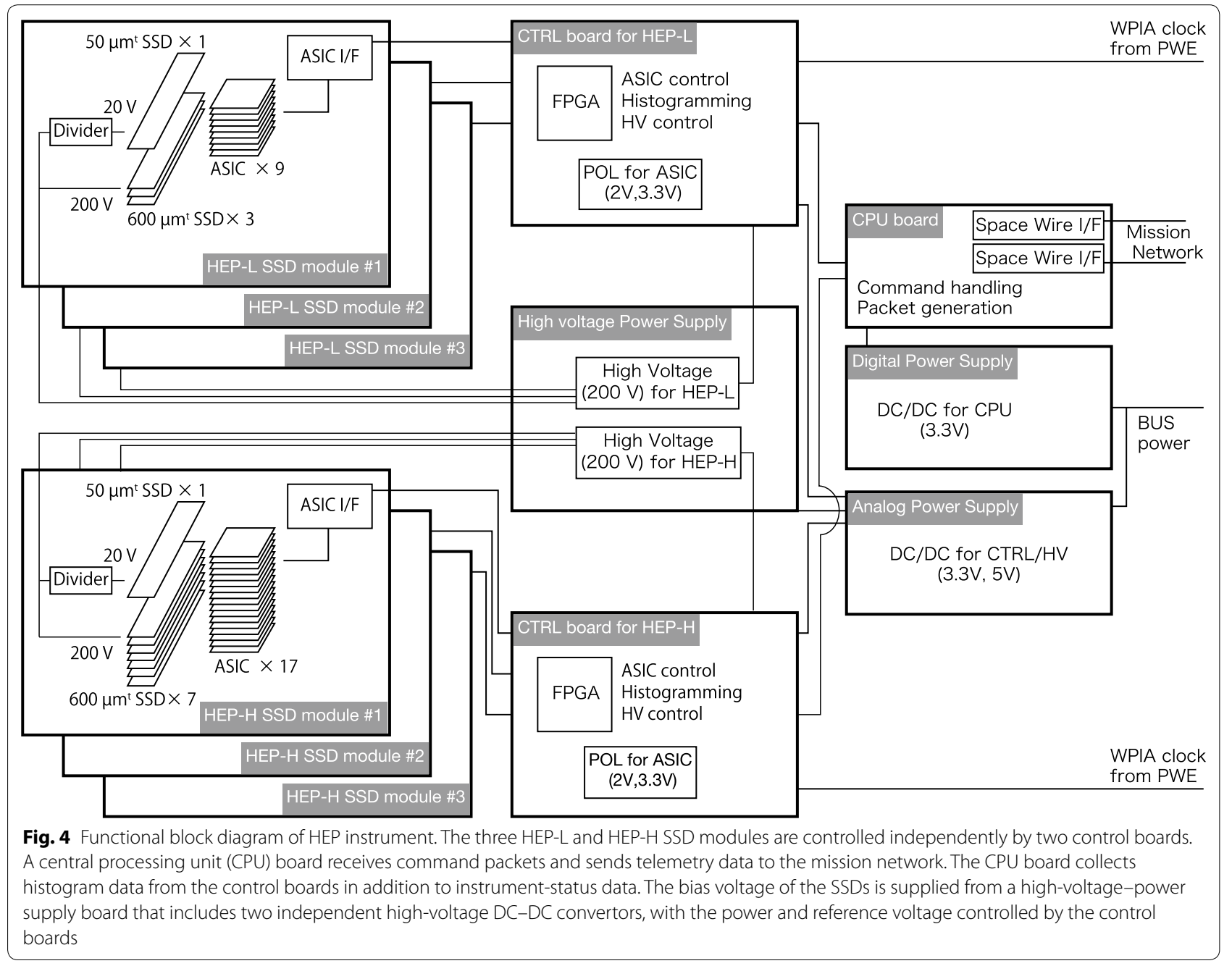



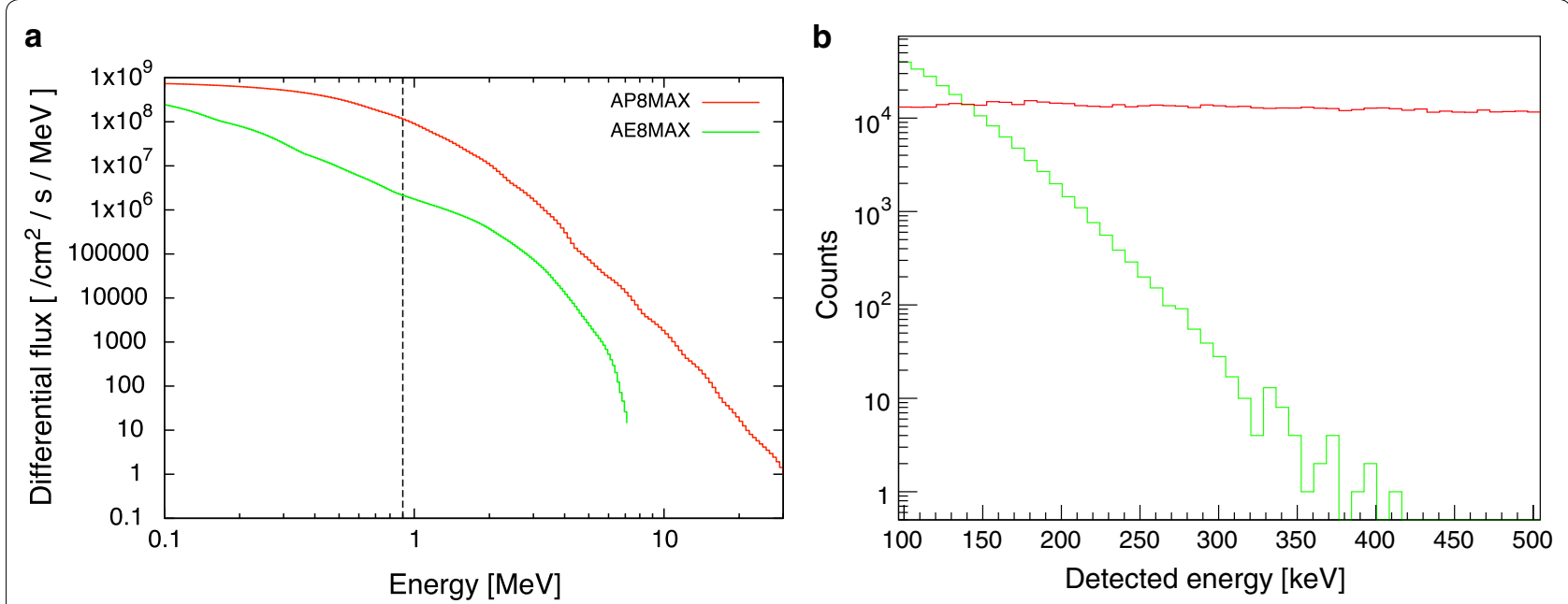

Fig. 5 a Differential fluxes of electrons and protons at $L=3$ based on AE8 MAX and AP8 MAX models; $\mathbf{b}$ detected energy spectra in first layer of stacked SSD when flux shown in a irradiates stacked SSDs of HEP- $L$

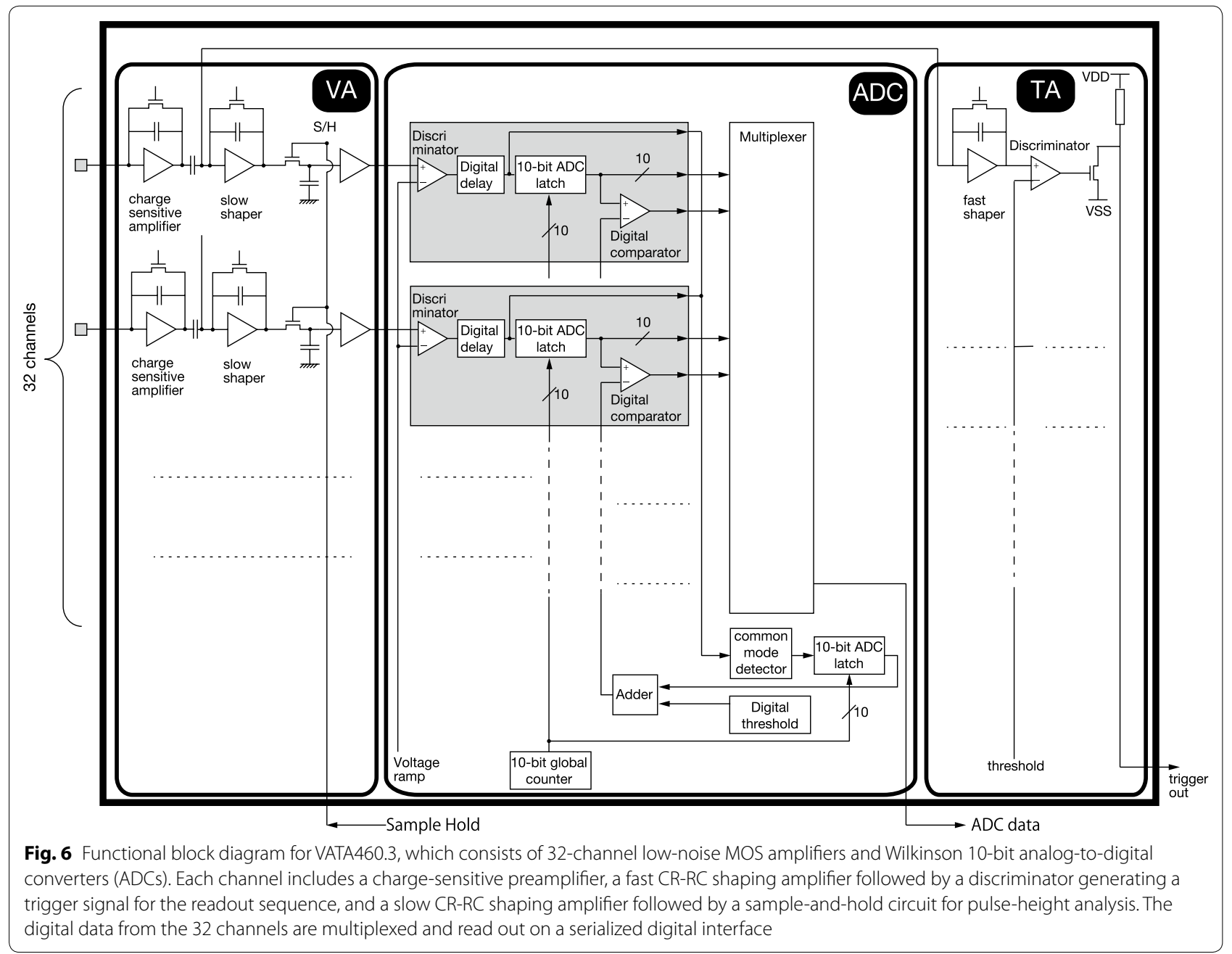


by disabling the triggers, which resulted to a reduced G-factor.

\section{Data processing and observation modes}

When the control board receives a trigger signal from one of the SSD modules, it issues a sample/hold signal to the ASICs and has them convert the analog data to digital data. Then, the control board reads out the digital data from all of the ASICs. Subsequently, the control board converts the ADC outputs to energy values and adds the contributions from all layers in order to calculate the total deposited energy. The detailed signal processing of an event is illustrated in Fig. 7 and is as follows: a trigger signal is issued if more than one of the outputs from the fast shapers in the ASICs of the first SSD layer is larger than the threshold. The control board sends a sample/hold signal to all of the ASICs with a defined delay after the triggering signal. The control board supplies the ADC clocks and receives a signal from the ASICs when the conversion has finished. After receiving these signals from all of the ASICs, the control board supplied the readout clocks of the ASICs. After reading out all of the data, the control board resets the digital part of the ASICs and waits for the next trigger signal. The total deposited energy and incident direction for each electron event are determined in this sequence. HEP generates telemetry data according to the operation mode and has the following four operation modes: setting mode, normal observation mode, S-WPIA observation mode, ASIC calibration mode, and event mode. In the setting mode,

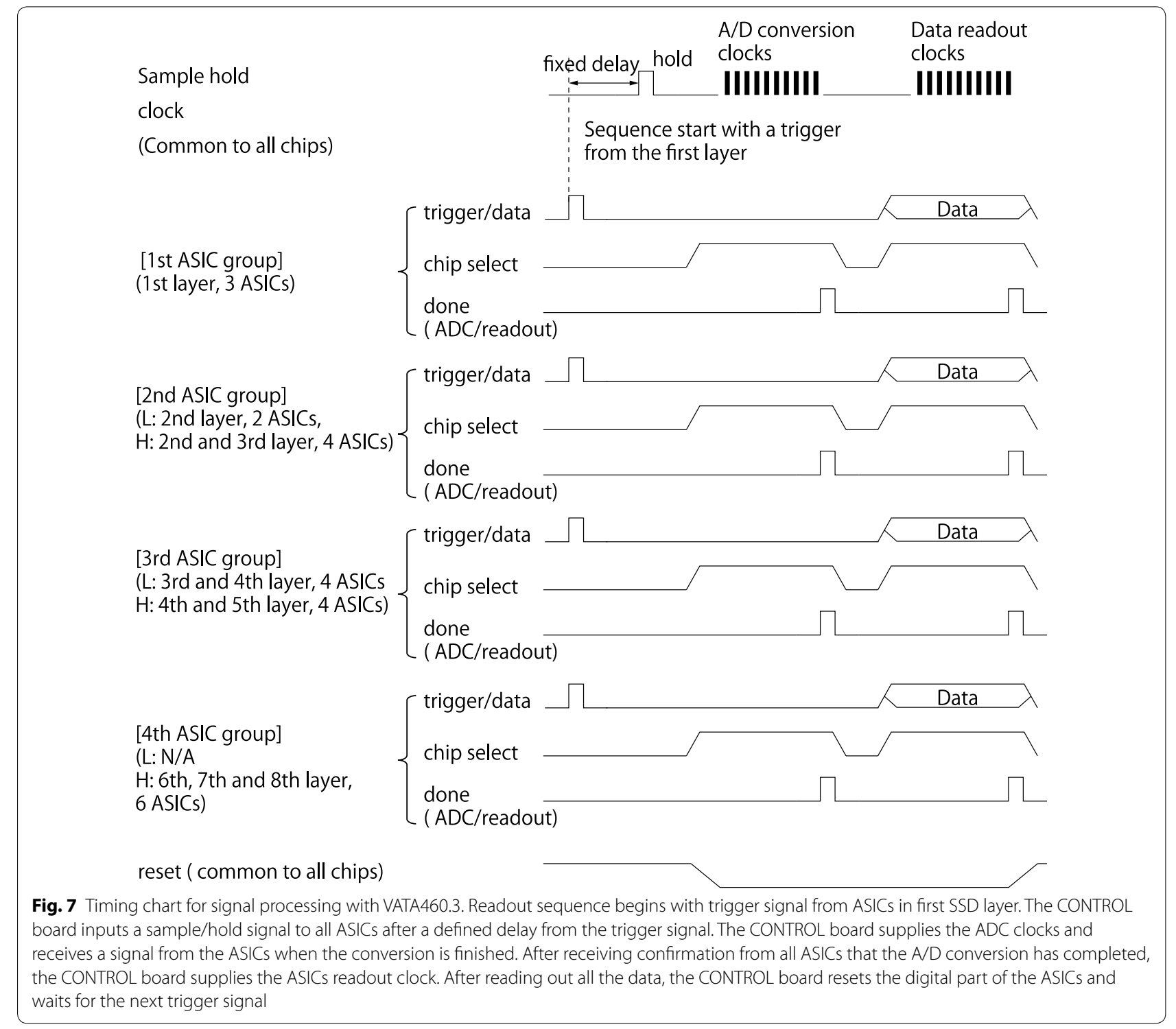


the ASIC control parameters, FPGA registers, and CPU software parameters can be set. Moreover, the ASIC control registers can only be set in this mode. In the normal observation mode, the energy histograms are generated onboard. Fifteen histograms corresponding to the channels shown in Fig. 2 are generated based on the deposit energy and incident elevation angle of each detected particle $(\theta$ in Fig. 2). The data accumulation continues for $1 / 16$ th of the satellite spin period, and the data are sent by the CPU software as telemetry data. Therefore, in normal observation mode, 240 histograms are generated for every spin. Each histogram has 16 energy bins, as listed in Table 1. In the S-WPIA observation mode, the packets for the S-WPIA are produced in addition to the normal observation mode data. The energy, incident direction, and timing information of each electron are sent to the mission data recorder. The prime objective of the S-WPIA is to measure the energy exchanged between the whistler-mode chorus emissions and the energetic electrons in the inner magnetosphere (Katoh et al. 2018). By assuming that $10 \mathrm{kHz}$ is the highest electron cyclotron frequency along the satellite's orbit at the equator, the wave period of the chorus emissions is found to be approximately $100 \mu \mathrm{s}$. A detection accuracy greater than $10 \mu \mathrm{s}$ can resolve the wave phase in the order of tens of degrees. To achieve this accuracy, HEP receives the S-WPIA clock signals at $524.288 \mathrm{kHz}(1.9-\mu \mathrm{s}$ period) from the plasma wave experiment (Kasahara et al. 2018b) instrument and has a counter module to count the clock signals. When HEP receives a trigger signal from the SSD modules, it latches the counter and adds the value to the S-WPIA data in order to achieve time indexing at a resolution of $1.9 \mu \mathrm{s}$. In the ASIC calibration mode, the waveforms of all ASIC channel shaping amplifier outputs are produced with test pulses. The raw ASIC data can be obtained in the event mode, and all individual event ASIC channel data are sent as telemetry data, for detailed calibration purposes. However, only a small portion of the triggering data can be relayed because the available telemetry data are limited.

In accordance with the ERG science observation plans, in the normal observation phase of ERG, HEP operates in the normal observation mode by default and enters the S-WPIA mode several times during a one-orbit revolution.

\section{Preflight testing}

For the energy calibration of all SSD channels, we tested the instrument with radioactive isotopes. We measured the energy spectra of all strips by using $\gamma$-ray sources placed in front of each SSD module. Figure 8 shows the spectrum of one SSD channel. From the center of the $60-\mathrm{keV} \gamma$-ray peak shown in this figure, the gain was

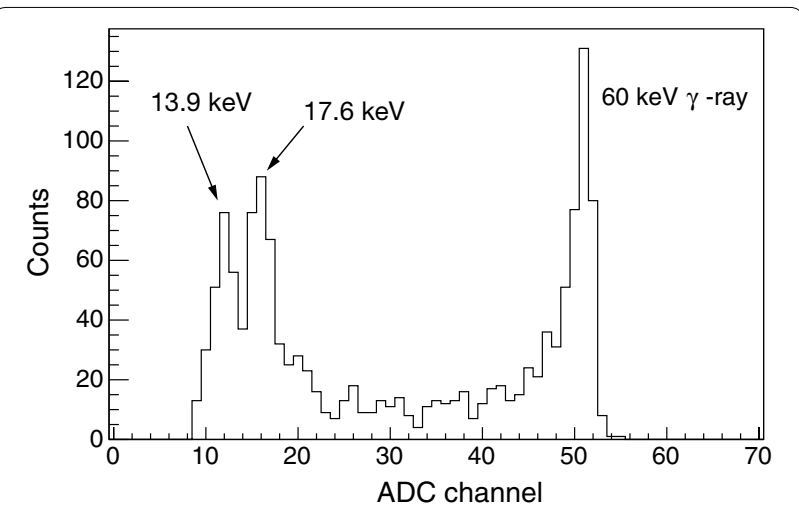

Fig. 8 -ray spectrum obtained by HEP SSD recorded with ${ }^{241} \mathrm{Am}$ radioactive isotope

calculated in order to serve as the conversion factor of the ADC to return energy values. Figure 9 shows the gains of all ASIC channels. HEP-L and HEP-H have 864 and 1632 channels, respectively (nine ASICs per HEP-L module and 17 ASICs per HEP-H module). Channels 0-95, 288-384, and 576-672 for HEP-L, and channels 0-95, 544-640, and 1088-1184 for HEP-H correspond to the $50-\mu \mathrm{m}$-thick SSD in the first layer of the stacked detectors. Because the deposited energy is expected to be small in the $50-\mu \mathrm{m}$-thick SSD, the parameters to control the ASIC A/D conversion are set such as needed to obtain a higher ADC value than that of the $600-\mu \mathrm{m}$-thick SSD. The variation of the gains is caused by the individual ASIC characteristics. In the first layer, the average gain of the ASICs is $0.92 \mathrm{ch} / \mathrm{keV}$, while that in the lower layers is $0.46 \mathrm{ch} / \mathrm{keV}$. Additionally, the standard deviations are 4.5 and $2.9 \%$, respectively. With these settings, the ASICs of the first layer and those of the lower layers cover the incident energies up to 1 and $2 \mathrm{MeV}$, respectively. The SSD dead layer is less than $3 \mu \mathrm{m}$, where the $70-\mathrm{keV}$ electrons lose $2.9 \mathrm{keV}$ (according to ESTAR). For the HEP requirements, this effect can be ignored.

The overall performance of the HEP was tested with electron beams from a particle accelerator, and a singleended Pelletron system (National Electrostatics Corporation, Model 6SH) at JAXA's Tsukuba Space Center (see the webpage of Space environment measurement laboratory at JAXA). The beam intensity ranges from 1 fA to $10 \mathrm{nA}$, and the system can generate electrons with energies of 0.4-2.0 MeV. The HEP was placed in a vacuum chamber such that the beam direction was parallel to the $Y_{\mathrm{sc}} Z_{\mathrm{sc}}$-plane marked in Fig. 1. Then, it was irradiated with monoenergetic electron beams. By rotating the HEP instrument around the $X_{s c}$-axis in the chamber, all SSD modules were irradiated with the beam and the detected energies and angular responses were recorded. The beam intensity was tuned such that the count rates 

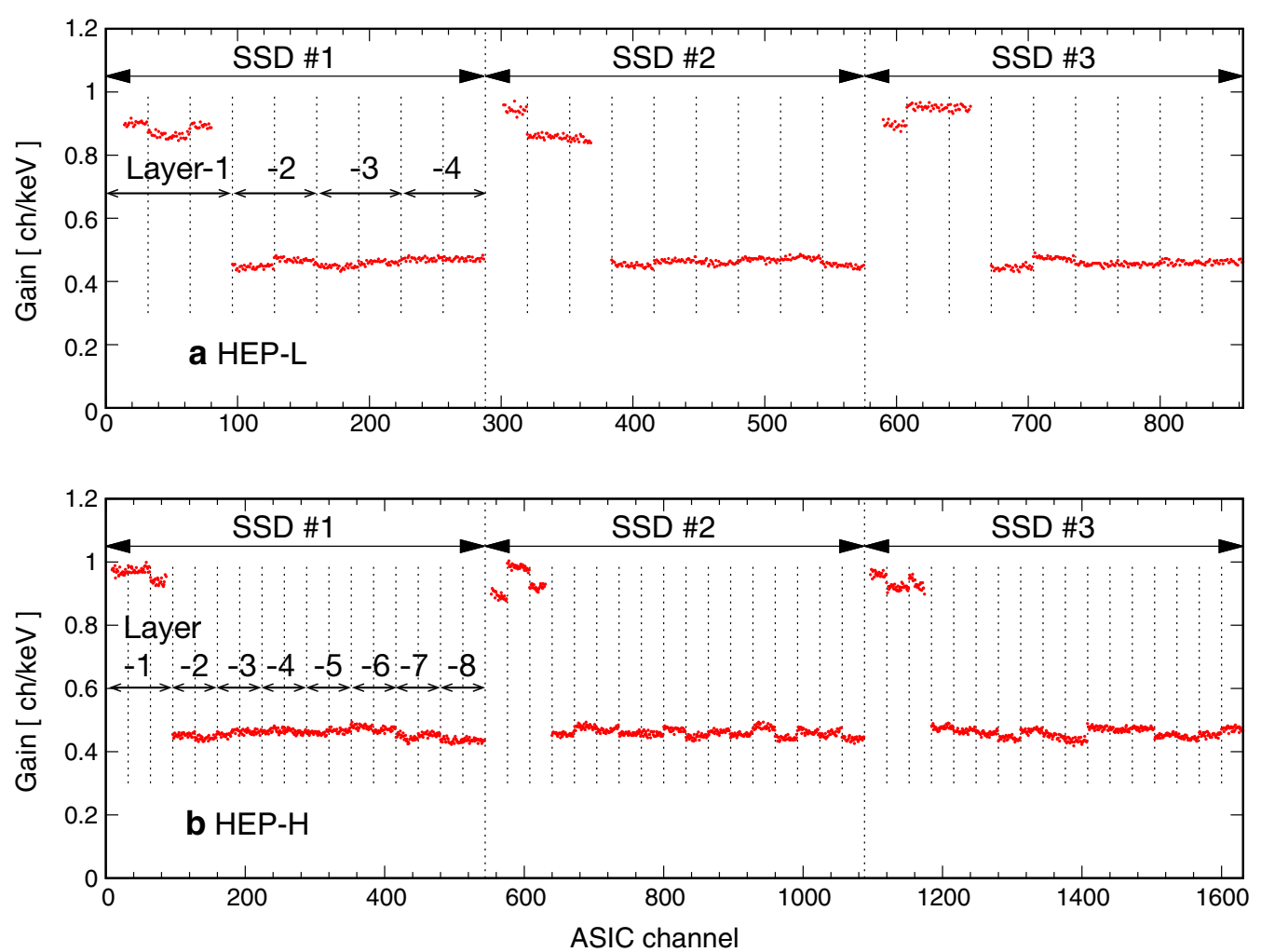

Fig. 9 VATA460.3 gain of all a HEP-L and $\mathbf{b}$ HEP-H channels of SSD modules. The dotted vertical lines indicate the first channel of each ASIC. The strips in the first layer are read out with three ASICs, and those in the lower layer are read out with two ASICs per layer. Therefore, 9 and 17 ASICS are used in the HEP-L and HEP-H SSD modules, respectively. Channels 0-95, 288-384, and 576-672 of HEP-L, and channels 0-95, 544-640, and 1088-1184 of HEP-H correspond to the first layer, which is the $50-\mu$ m-thick SSD
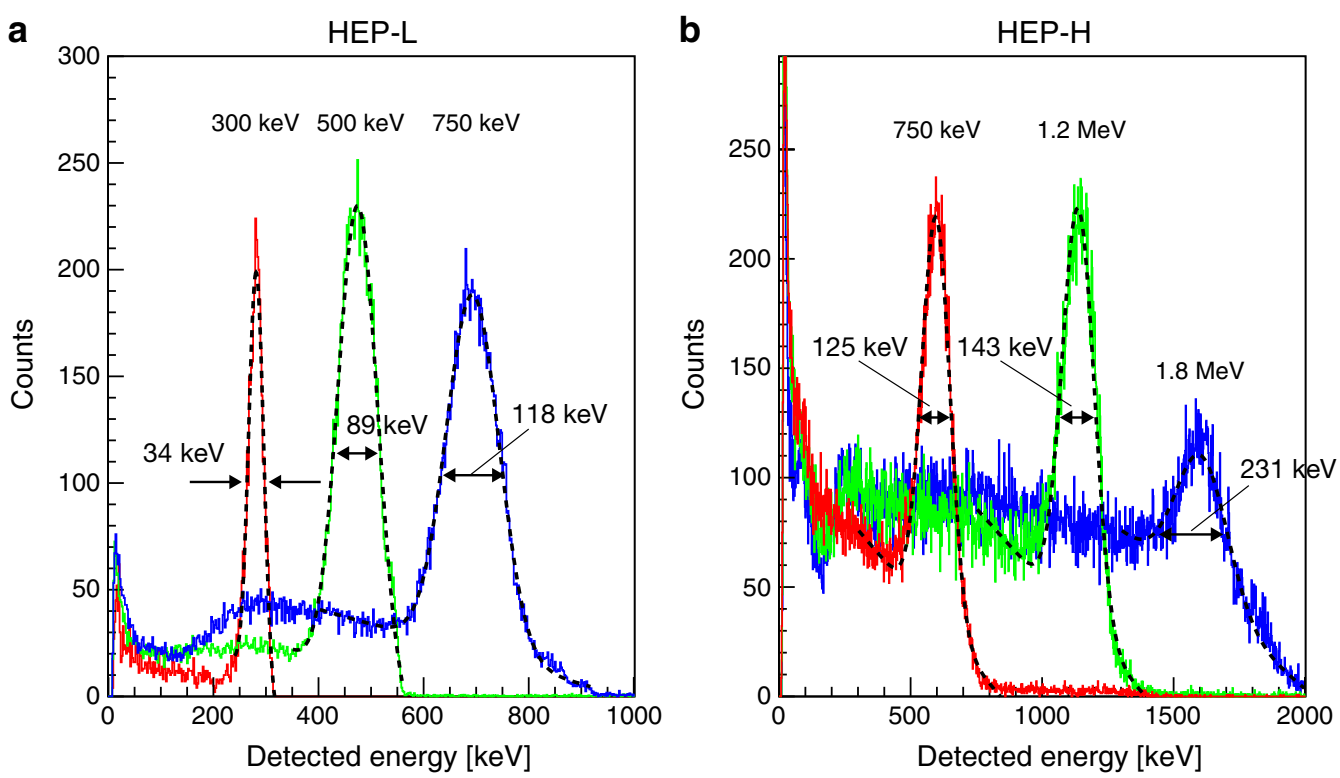

Fig. 10 Electron spectra measured with HEP flight model. a Spectra detected by HEP-L show electrons with 300, 500, and 750 keV of energy. b Spectra detected by HEP-H show electrons with energy of $750 \mathrm{keV}, 1.2 \mathrm{MeV}$, and 1.8 MeV. Dotted line shows result of fitting with Gaussian function in addition to straight line 
for the SSD module were approximately a few hundred $\mathrm{Hz}$. Figure 10 shows the energy spectra obtained with the HEP flight model when the input electron energy was 300,500 , and $750 \mathrm{keV}$ for HEP-L, and $750 \mathrm{keV}$, 1.2 MeV, and $1.8 \mathrm{MeV}$ for HEP-H. In these tests, we used the electron beam with a normal incidence to the SSDs. The triggers from the strips in the first layer were enabled, and the total deposited energy was determined by summing up the contributions of all layers. The spectral peaks were fitted with a Gaussian function in addition to a straight line. The full-width at half maximum (FWHM) values were $34 \mathrm{keV}$ at $300 \mathrm{keV}, 89 \mathrm{keV}$ at $500 \mathrm{keV}$, and $118 \mathrm{keV}$ at $750 \mathrm{keV}$ for HEP-L, while those for HEP-H were $125 \mathrm{keV}$ at $750 \mathrm{keV}, 143 \mathrm{keV}$ at $1.2 \mathrm{MeV}$, and $231 \mathrm{keV}$ at $1.8 \mathrm{MeV}$. An energy resolution of less than $20 \%$ was realized. According to ESTAR, the energy loss at the $300-\mu$ m-thick $\mathrm{Al}$ sheet of HEP-H is $0.12 \mathrm{MeV}$ for a $0.7-2 \mathrm{MeV}$ electron. The difference between the energy of the input electrons and the detected energy was consistent with the energy loss. Moreover, the loss should be considered when we infer the incident energy of the electrons from the detected signals, and the low-energy tails in the spectra should also be considered. To evaluate the incident-angle response, the HEP instrument was rotated around the $X_{\mathrm{sc}}$-axis marked in Fig. 1. The measured angular responses when changing the beam incident angle are shown in Fig. 11. The figure shows the histograms that resulted from the measurements with seven and three different incident angles for HEP-L and
HEP-H, respectively. To evaluate the angular resolution, the histograms had more bins than the azimuthal channels in Fig. 2, while the histograms of HEP-L and HEP-H had 60 bins and 12 bins covering $60^{\circ}$, respectively. Thus, the bin width of the HEP-L and HEP-H responses were $1^{\circ}$ and $3^{\circ}$, respectively. These values are smaller than the angle uncertainty caused by the position resolution, which is estimated based on the detector simulation described above. Based on the responses, the angular resolution had a FWHM of approximately $4^{\circ}$ (4 bins) and $15^{\circ}$ (5 bins) for HEP-L and HEP-H, respectively.

\section{In-orbit operation and flight performance}

On February 2, 2017, HEP was turned on for the first time while in orbit, and the initial checkout was successful. The bias voltage of the silicon detectors was limited below $50 \mathrm{~V}$, and the count rates were monitored for several days.

On February 6, 2017, a nominal bias voltage of $200 \mathrm{~V}$ was applied, and the detector performance was tested and found to be normal. We checked the noise level and waveform of the shaping amplifier output for every channel. The waveforms of several channels are shown in Fig. 12, alongside the waveforms measured before launch, for comparison purposes. The peak times and pulse heights were not different before and after the launch.

After the other instruments aboard the ERG had finished the initial checkouts followed by the verification of the overall operation plan, the ERG satellite was shifted
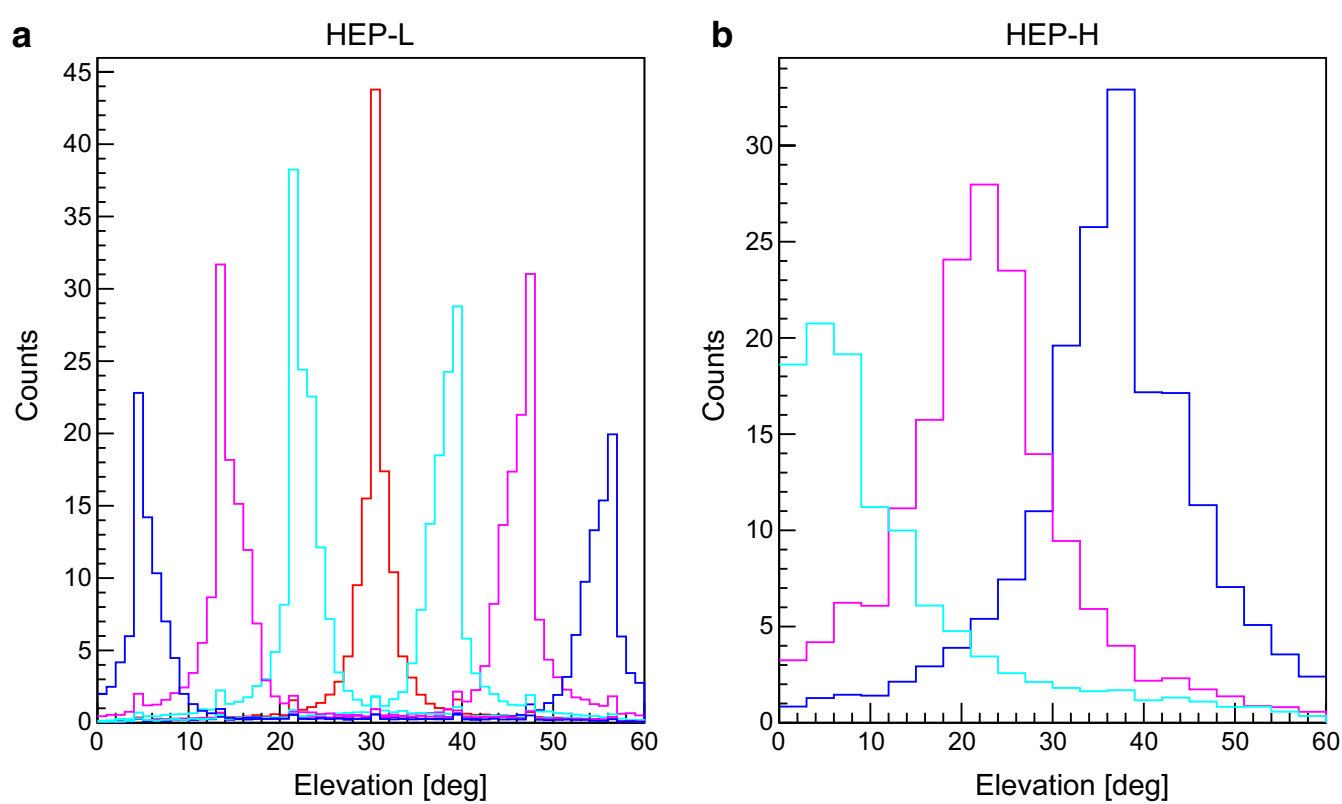

Fig. 11 Angular response measured with HEP flight model. a Angular responses measured while changing the beam incident angle on HEP-L (i.e., rotating HEP around $X_{s c}$-axis in Fig. 1); bin width is $1^{\circ}$. b Angular responses measured when changing beam incident angle on HEP-H (i.e., rotating HEP around $X_{\text {sc }}$-axis in Fig. 1); bin width is $3^{\circ}$ 

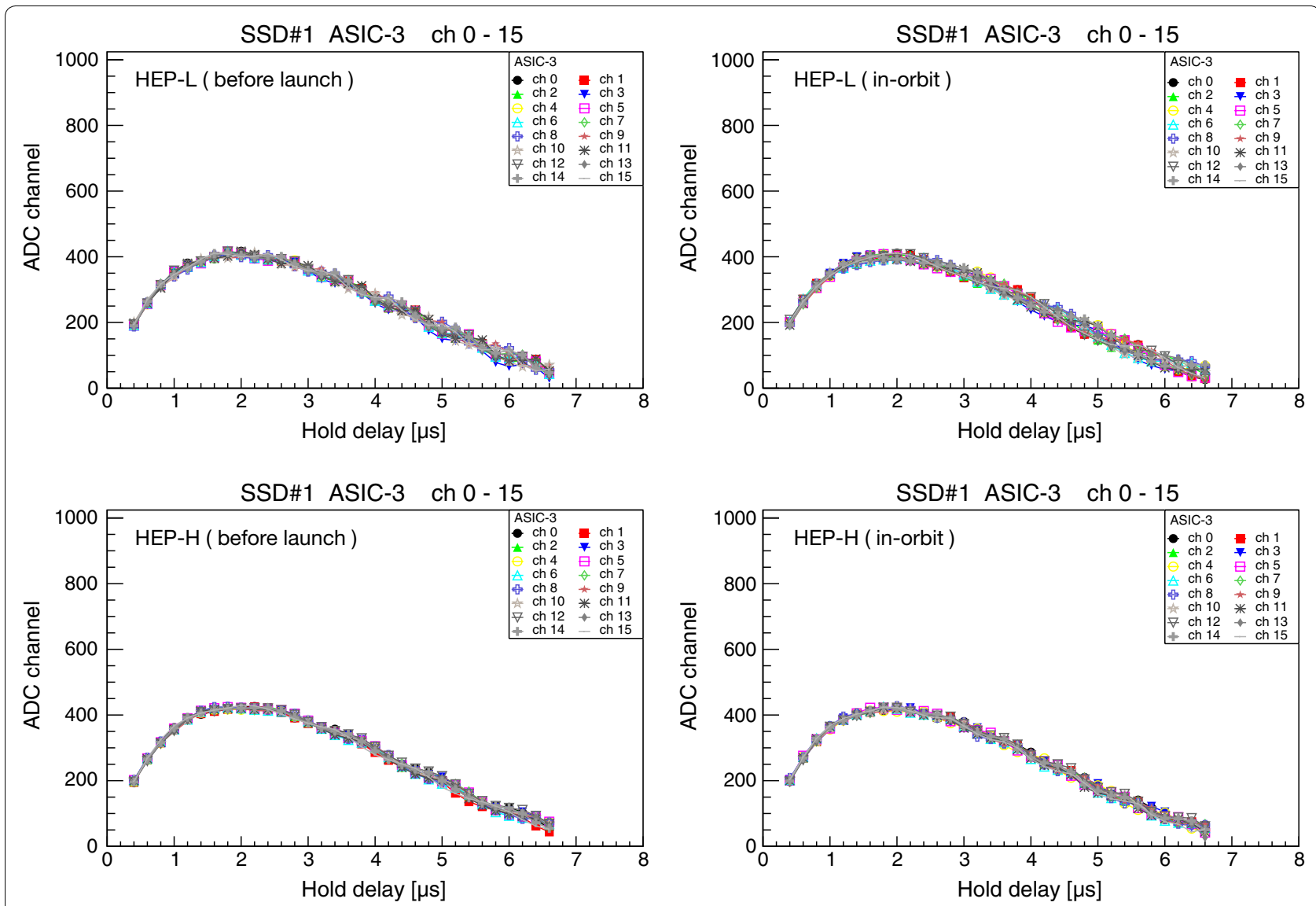

Fig. 12 Waveforms of shaping amplifier output measured while in orbit (right) compared to preflight measurements (left) for performance verification

to its normal observation phase in late March 2017. Since March 16, 2017, HEP has been operating in the normal observation mode, and several parameters controlling the channels triggered by the SSDs have been changed. Figure 13 shows the energy-time $(E-t)$ spectrograms, which represent the change in the time of the spinaveraged count rate observed by HEP with the L-value, which is the radial distance of the electron drift orbits in the magnetic equatorial plane, and is measured in units of Earth radii. The E-t spectrograms in Fig. 13 cover 2 days and five orbital revolutions. As the satellite moves through different $L$-values, the observed count rates change and peak at $L=4-5$, which corresponds to the outer radiation belt, as expected. Near the orbit's perigee $(L=3)$, HEP ceases the observation because of the high count rates of high-energy protons. The counts measured with HEP-L in the vicinity of $0.9 \mathrm{MeV}$ increase near the perigee because of high-energy protons.

\section{Summary and future work}

The HEP instrument has successfully begun the observation of electrons with energies of $70 \mathrm{keV}-2 \mathrm{MeV}$ in the
Earth's inner magnetosphere. The HEP consists of three HEP-L modules and three HEP-H modules. HEP-L detects $70 \mathrm{keV}-1 \mathrm{MeV}$ electrons and has a maximum G-factor of $9.3 \times 10^{-4} \mathrm{~cm}^{2}$ sr, while HEP-H observes $0.7 \mathrm{MeV}-2 \mathrm{MeV}$ electrons and has a maximum G-factor of $9.3 \times 10^{-3} \mathrm{~cm}^{2} \mathrm{sr}$ at maximum. These modules are pin-hole cameras consisting of mechanical collimators and SSDs. The signals from a total of 2355 strips are processed by 78 readout ASICs. Before the satellite was launched, all channels were evaluated with reference signals from radioactive isotopes and the overall HEP performance was evaluated with electron beams. In orbit, the waveforms of the calibration pulses indicated that the HEP functioned properly after it was launched. From the initial results of the energy-time spectrograms, the HEP recorded high electron count rates in the outer radiation belt.

A simulation of the detector is under development in order to convert the count data to physical quantities with higher precision. We will model the HEP detector geometry and particle interaction with the detectors and surrounding materials by utilizing the Geant4 library. 


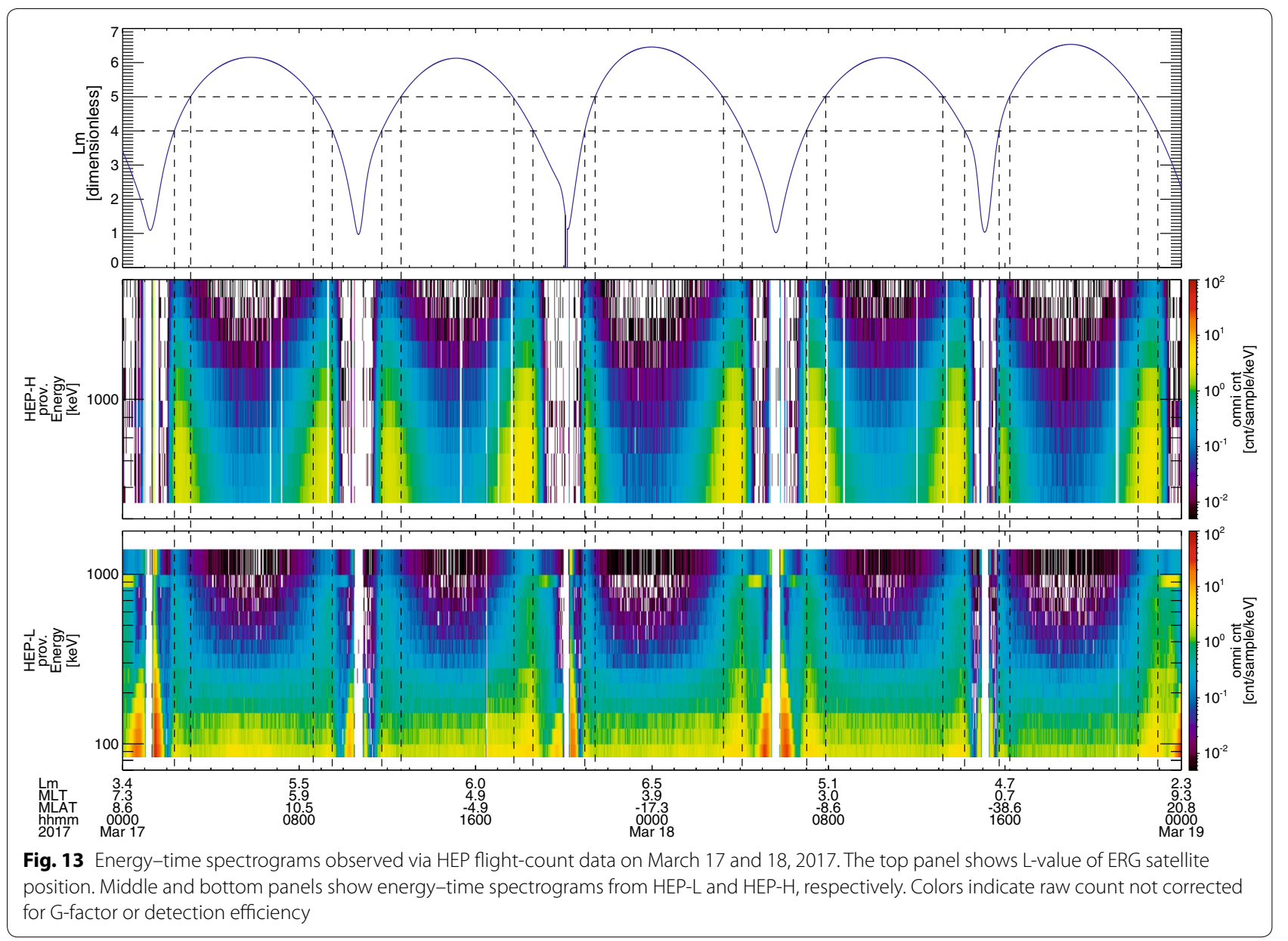

After the validation tests of the simulator and the comparisons between the simulation results and the experimental results, we will be able to calculate the detector response by using the simulator. As shown in Fig. 10, monoenergetic electrons can be detected in lower energy channels. A spectrum detected in orbit is the superposition of signals from electrons with different energy. By using the simulator, we can estimate how many of the detected counts in the lower energy channels are contributed by higher-energy electrons, and thereby determine the incident flux with higher precision. Thus, we will be able to deduce the distribution of incident electrons with higher precision from the direction and energy detections in orbit. A detailed report regarding the simulator and its validation will be published in the future.

\section{Authors' contributions}

TM led the design and development of HEP.TT supported and offered advice during all phases of design and development. SK provided information necessary to the development with regard to the instruments of the ERG mission. WM worked on the basic development of the VATA460 series. MH worked on the initial collimator design and stacked SSD module. All authors read and approved the final manuscript.

\section{Author details}

${ }^{1}$ Institute of Space and Astronautical Science, Japan Aerospace Exploration Agency, Sagamihara 252-5210, Kanagawa, Japan. ${ }^{2}$ Department of Earth and Planetary Science, School of Science, The University of Tokyo, Bunkyo-ku 113-0033, Tokyo, Japan. ${ }^{3}$ Department of Aeronautics and Astronautics, Tokai University, Hiratsuka-shi 259-1292, Kanagawa, Japan. ${ }^{4}$ Institute of Space-Earth Environmental Research, Nagoya University, Nagoya 464-8601, Aichi, Japan.

\section{Acknowledgements}

We would like to thank all members of the ERG project for their long-lasting efforts to realize this mission. The HEP instrument was developed by Mitsubishi Heavy Industries Co, Ltd. The high-voltage board in HEP was developed by Meisei Electric Co., Ltd. The silicon detectors were manufactured by Hamamatsu Photonics K. K., and VATA460.3 was manufactured by IDEAS, in Norway. The development of VATA460.3 has profited from the design heritage of BepiColombo MMO and Astro-H SGD/HXI. From the Astro-H team, we would like to thank S. Watanabe and M. Kokubun in particular, for their support and sharing of experience. The electron beam facility used in the calibration of HEP is maintained by the Research and Development Directorate in JAXA. Quick plots are distributed by the ERG science center, which also makes level-2 data available. We also thank F. Makino, T. Goka, H. Tajima, and T. Mukai, who reviewed the design and development of HEP during each phase of its development.

\section{Competing interests}

The authors declare that they have no competing interests. 
Availability of data and materials

The data and materials used in this research are available from the corresponding author upon reasonable request.

\section{Consent for publication}

Not applicable.

Ethics approval and consent to participate

Not applicable.

\section{Funding}

The ERG (Arase) project is funded by ISAS/JAXA.

\section{Publisher's Note}

Springer Nature remains neutral with regard to jurisdictional claims in published maps and institutional affiliations.

Received: 4 October 2017 Accepted: 25 April 2018

Published online: 08 May 2018

\section{References}

Baker DN, Blake JB, Klebesadel RW, Higbie PR (1986) Highly relativistic electrons in the earth's outer magnetosphere: 1. Lifetimes and temporal history 1979-1984. J Geophys Res 91(A4):4265-4276. https://doi. org/10.1029/JA091iA04p04265

Hikishima M, Kojima H, Katoh Y, Kasahara Y, Kasahara S, Mitani T, Higashio N, Matsuoka A, Miyoshi Y, Asamura K, Takashima T, Yokota S, Kitahara M, Matsuda S (2018) Data processing in the software-type wave-particle interaction analyzer on board the Arase satellite. Earth Planets Space 70:80. https://doi.org/10.1186/s40623-018-0856-y

Katoh Y, Kojima H, Hikishima M, Takashima T, Asamura K, Miyoshi Y, Kasahara Y, Kasahara S, Mitani T, Higashio N, Matsuoka A, Ozaki M, Yagitani S, Yokota S, Matsuda S, Kitahara M, Shinohara I (2018) Software-type wave-particle interaction analyzer on board the Arase satellite. Earth Planets Space 70:4. https://doi.org/10.1186/s40623-017-0771-7

Kasahara S, Yokota S, Mitani T, Asamura K, Hirahara M, Shibano Y, Takashima T (2018a) Medium-energy particle experiments-electron analyser (MEP-e) for the energization and radiation in geospace (ERG) mission. Earth Planets Space 70:69. https://doi.org/10.1186/s40623-018-0847-z

Kasahara Y, Kasaba Y, Kojima H, Yagitani S, Ishisaka K, Kumamoto A, Tsuchiya F, Ozaki M, Matsuda S, Imachi T, Miyoshi Y, Hikishima M, Katoh Y, Ota M, Shoji M, Matsuoka A, Shinohara I (2018b) The plasma wave experiment (PWE) on board the Arase (ERG) satellite. Earth Planets Space 70:86. https ://doi.org/10.1186/s40623-018-0842-4
Kazama Y, Wang BJ, Wang SY, Ho PTP, Tam SWY (2017) Low-energy particle experiments - electron analyzer onboard the Arase spacecraft. Earth Planets Space 69:165. https://doi.org/10.1186/s40623-017-0748-6

Makishima K (1999) Energy non-equipartition processes in the Universe. Astron Nachr 320:163-166

Miyoshi Y, Ono T, Takashima T, Asamura K, Hirahara M, Kasaba Y, Matsuoka A, Kojima H, Shiokawa K, Seki K, Fujimoto M, Nagatsuma T, Cheng CZ, Kazama Y, Kasahara S, Mitani T, Matsumoto H, Higashio N, Kumamoto A, Yagitani S, Kasahara Y, Ishisaka K, Blomberg L, Fujimoto A, Katoh Y, Ebihara Y, Omura Y, Nose Hori T, Miyashita Y, Tanaka Y-M, Segawa TT, ERG working group (2012) The Energization and Radiation in Geospace (ERG) project. In: Summers D, Mann IR, Baker DN, Schulz M (eds) Dynamics of the earth's radiation belts and inner magnetosphere. American Geophysical Union, Washington. https://doi.org/10.1029/2012GM001304

Nagai T (1988) Space weather forecast: prediction of relativistic electron intensity at synchronous orbit. Geophys Res Lett 15:425. https://doi. org/10.1029/GL015i005p00425

Reeves GD, McAdams KL, Friedel RHW, O'Brien TP (2003) Acceleration and loss of relativistic electrons during geomagnetic storms. Res Lett, Geophys. https://doi.org/10.1029/2002GL016513

Takashima T, Ogawa E, Asamura K, Hikishima M (2018) Design of a mission network system using SpaceWire for scientific payloads onboard the Arase spacecraft. Earth Planets Space 70:71. https://doi.org/10.1186/ s40623-018-0839-Z

Saito Y, Sauvaud JA, Hirahara M, Barabash S, Delcourt D, Takashima T, Asamura K, BepiColombo MMO, Team MPPE (2010) Scientific objectives and instrumentation of Mercury Plasma Particle Experiment (MPPE) onboard MMO. Planet Space Sci 58:82-200. https://doi.org/10.1016/j.pss.2008.06.003

Watanabe S, Tajima H, Fukazawa Y et al (2014) The Si/CdTe semiconductor Compton camera of the ASTRO-H Soft Gamma-ray Detector (SGD). Nucl Instrum Methods Phys Res A 765:192. https://doi.org/10.1016/j. nima.2014.05.127

\section{Online databases}

AE-8/AP-8 Radiation Belt Models. https://ccmc.gsfc.nasa.gov/modelweb/ models/trap.php

Geant4 toolkit. http://geant4.cern.ch

Space environment measurement laboratory, JAXA. http://sees.tksc.jaxa.jp/ fw_e/dfw/SEES/English/Labo/labo_e.shtml

Stopping-Power \& Range Tables for Electrons, Protons, and Helium lons, National Institute of Standards and Technology, U.S. Department of Commerce. https://www.nist.gov/pml/stopping-power-range-tables-elect rons-protons-and-helium-ions

\section{Submit your manuscript to a SpringerOpen ${ }^{\odot}$ journal and benefit from:}

- Convenient online submission

- Rigorous peer review

- Open access: articles freely available online

- High visibility within the field

- Retaining the copyright to your article

Submit your next manuscript at $\mathbf{s p r i n g e r o p e n . c o m ~}$ 